\title{
Multiparameter mathematical models of the filtration problem of unstructured and structured fluids
}

\author{
Shukur Qayumov ${ }^{1 *}$, Arslan Mardanov ${ }^{1}$, Anvar $_{\text {Qayumov }}{ }^{2}$, and Tojiboy Xaitov ${ }^{1}$ \\ ${ }^{1}$ Tashkent State Technical University, Tashkent, Uzbekistan \\ ${ }^{2}$ Inha University in Tashkent city, Tashkent, Uzbekistan
}

\begin{abstract}
The article is devoted to constructing a generalized mathematical model and the method of their solution of the process of filtration of fluids with various linear and nonlinear characteristics. The multiparameter model contains 13 mathematical models developed in due time by scientific research and included new mathematical models. The classification of these models is carried out in accordance with the laws of filtration, and they are confirmed by the results of numerical solutions. For unknown boundaries of disturbances, the application of the "shuttle" iteration method made it possible to reduce the number of iterations.
\end{abstract}

\section{Introduction}

It is known that the main reserves of fluids (oil, gas, gas condensate, and water) are contained in underground porous media. Therefore, the study of the filtration process of these fluids is very important and has been carried out for a long time. Researchers in these areas have published numerous scientific articles and monographs. Sometimes from the outside, it seems that everything related to the problem, the development of fields in porous media, has been studied, and there are no "white spots" in this area.

However, science is interesting in that the researcher always finds unexplored or littleexplored areas. Each researcher, studying a specific problem, contributes his rounders to the developed and collected knowledge bases by other previous researchers. The applicant, studying the accumulated knowledge, always finds unexplored points in which there are gaps and begins to develop them, exploring from different angles and in detail. This is how science is born and develops.

Take a close look at the state of knowledge of the existing and completed studies in the direction (area) of fluid movement in porous media. You can see that this study was carried out (we will denote conditionally) for unstructured, structured, and abnormally structured fluids. Unstructured fluids are those fluids whose motion is described by Darcy's laws and their varieties. You can also attach filtering tasks with nonlinear effects to them. Structured fluids are those fluids that belong to one component. Still, they have different characteristics and in their physical characteristics that, when the stress changes as a result of the internal interacting forces of the fluid itself, as well as the geological - mineralogical

*Corresponding author: kayumovmatemic@gmail.com 
structure of the skeleton of the porous medium, behave differently. Therefore they can be called as structured fluids [1]. For fluids and a medium, this property is characteristic of a destroyed, little destroyed, undisturbed structure; the behavior differs from the movement of unstructured ones, which greatly affects the mobility and filtration rate. These zones are a zone of low mobility or a creep zone where the structure of the fluid is practically not destroyed, a zone of medium (similar to abnormal) mobility, where the relationship between the fluid velocity and the pressure gradient is nonlinear, the zone of maximum mobility, where the relationship between the velocity and the pressure gradient is almost linear (with weight coefficients) [2]. Anomalously structured fluids can be attributed to the filtration process of those types of structured fluids, where nonlinear anomalous effects are present in zones of low mobility, in zones of nonlinear mobility, and zones of increased mobility.

Their critical values of the pressure gradient can exist between these zones. The mathematical models of these problems differ from the models of elastic, viscoelastic, elastically plastic, and other available models of fluid filtration in porous media.

Investigations related to the mathematical modeling of these areas were carried out in works [3-9] in one-dimensional and two-dimensional cases in one reservoir porous media, where separate models and combined mathematical models were proposed. When studying the problem of filtration of anomalous oil in [10], a transformed fractional flow function was used, reflecting the rheological features of the flow. The structure of behavior of nonNewtonian fluids in a porous medium was studied and numerically investigated in $[11,12]$. When deriving a mathematical model of fluid filtration, the structure of the model depends on the assumptions of the method for approximating the functional relationship with the fluid velocity and pressure gradient. Geometrically, it corresponds to different curves, and each curve describes a particular filtration law; and following this, a mathematical model of the problem can be built. Many of these curves correspond to many mathematical models, each of which is studied separately by researchers.

The combination of these models leads to the creation (construction) of multiparameter models. It makes it possible to write a single parameterized mathematical model with parameterized boundary and boundary conditions.

In [13], an approximately analytical solution was constructed for a one-dimensional problem of structured fluids, and an iterative computational algorithm for the solution was developed for a two-dimensional problem. The work $[14,15]$ is devoted to constructing a multiparameter model for the problem of one-dimensional filtration of unstructured and anomalously structured fluids, where 9 filtration laws were included in the model.

The problem of three-dimensional filtration of non-Newtonian fluids when the motion of fluids obeys a curvilinear law [16] was studied and investigated in [17], and a computational algorithm for the solution was constructed. Further generalization of multiparameter problems, which include all existing and possibly new types of approximation of the functional dependence between the filtration rate and pressure gradient, is a necessary and important element in the theory of building mathematical models, which are universal multiparameter initial boundary value problems as in the theory of filtration of unstructured, structured and anomalous structured fluids, and for problems of mathematical physics.

\section{Methods}

Let the porous medium (region $\Omega$ ) saturated with fluids of the above types each separately, and the formation pressure at the initial moment of time is also known. When the internal (boundary) sources start to work, the formation is disturbed, and, depending on the types of fluid, various zones are formed with their own movable unknown boundaries. The physical 
problem, in this case, is mathematically formulated as follows: It is necessary to find a continuous unknown function $u(x, t)$ and unknown moving boundaries from the following initial boundary value $l_{r}(t), R_{1 \sigma}(t), R_{2 \sigma}(t)$ problem:

$$
\begin{gathered}
\frac{\partial}{\partial x}\left(x^{\tilde{\alpha}} \frac{h k(x)}{\mu} A\left(\frac{|\nabla u|}{\beta}\right)\right) \frac{\partial u}{\partial x}=M(x, t, u) \frac{\partial u}{\partial t}+f(x, t), \\
x \in \Omega, t>0, x_{0}<x<x_{L}
\end{gathered}
$$

with initial conditions

$$
u(x, 0)=u_{0}(x) . \quad x \in \bar{\Omega}
$$

boundary conditions

$$
\begin{aligned}
& \left.a_{1} \frac{x^{\tilde{\alpha}} h k(x)}{\mu b} A\left(\frac{|\nabla u|}{\beta}\right) \frac{\partial u}{\partial x}\right|_{x=x_{0}}+\left.b_{1} u(x, t)\right|_{x=x_{0}}=\varphi_{0}\left(x_{0}, t\right), t>0 \\
& \left.a_{2} \frac{x^{\tilde{\alpha}} h \cdot k(x)}{\mu b} A\left(\frac{|\nabla u|}{\beta}\right) \frac{\partial u}{\partial x}\right|_{x=x_{L}}+\left.b_{2} u(x, t)\right|_{x=x_{L}}=\varphi_{L}\left(x_{L}, t\right), t>0
\end{aligned}
$$

conditions for the existence of unknown moving boundaries:

$$
\begin{aligned}
& \left.e_{1} A\left(\frac{|\nabla u|}{\beta}\right)\right|_{x=l_{r}^{+}}=0, \quad e_{1}\left(\left.u(x, t)\right|_{x \in D}-u_{0}(x)\right)=0 \\
& x=l_{r}^{ \pm} \pm 0, r=\{0 ; 1\} ;\left(r=0, x=l_{0}^{+}+0, r=n, x=l_{n}^{-}-0\right) \\
& e_{2}\left(\left|\nabla u\left\|_{x=l_{r}^{\mp} \mp 0}=\mid \nabla u\right\|_{x=l_{r}^{\mp} \pm 0}=\beta\right), e_{2}\left(\left.u(x, t)\right|_{x=l_{r}^{\mp} \mp 0}-\left.u(x, t)\right|_{x=l_{r}^{\mp} \pm 0}\right)=0, \quad r=\overline{0, n}\right. \text {. } \\
& e_{3}\left(\mid \nabla u \|_{x=l_{r} \mp 0}-\beta\right)=0, \quad e_{3}\left(u(x, t)_{x \in D}-u_{0}(x)\right)=0 \\
& e_{4}\left(\left|\nabla u\left\|_{x=R_{1}^{ \pm} \sigma^{ \pm}}=\mid \nabla u\right\|_{x=R_{1}^{ \pm}+0}^{ \pm}=\beta_{1}\right), e_{4}\left(\left|\nabla u\left\|_{x=R_{2}^{ \pm} \sigma^{-0}}=\mid \nabla u\right\|_{x=R_{2}^{ \pm} \sigma^{+0}}=\beta_{2}\right)\right.\right. \\
& e_{4}\left(\left.u(x, t)\right|_{x=R_{1}^{ \pm} \sigma^{-0}}-\left.u(x, t)\right|_{x=R_{1}^{ \pm} \sigma^{+0}}\right)=0, e_{4}\left(\left.u(x, t)\right|_{x=R_{2}^{ \pm} \sigma^{-0}}-\left.u(x, t)\right|_{x=R_{2} \sigma^{+0}}\right)=0 \\
& e_{4}\left(\left|\nabla u\left\|_{x=R_{1 r}^{ \pm}-0}=\mid \nabla u\right\|_{x=R_{1 r}^{ \pm}+0}=\beta_{1}\right), e_{4}\left(\left|\nabla u\left\|_{x=R_{2 r}^{ \pm}-0}=\mid \nabla u\right\|_{x=R_{2 r}^{ \pm}+0}=\beta_{2}\right)\right.\right. \\
& \sigma=\{0 ; n\}, r=\overline{1, n-1} \text {. }
\end{aligned}
$$




$$
e_{4}\left(\left.u(x, t)\right|_{x=R_{1 r}^{ \pm}-0}-\left.u(x, t)\right|_{x=R_{1 r}^{ \pm}+0}\right)=0, e_{4}\left(\left.u(x, t)\right|_{x=R_{2 r}^{ \pm}-0}-\left.u(x, t)\right|_{x=R_{2 r}^{ \pm}+0}\right)=0
$$

Where

$$
A(\eta)=\frac{\left[\eta^{\lambda}-\gamma^{\lambda}(2-\lambda)\right]^{\theta} \cdot \eta^{-1} \cdot \beta^{\theta-1}}{\left\{1+\left[(\lambda-\gamma)^{\lambda}+\eta^{\lambda}\right]^{\frac{1}{2}}\right\}^{\lambda-1}}
$$

Parameterized function (12) parameters $\theta, \lambda, \gamma, \eta$ take values following table 1 and determines the specific form of this function for each filtration law.

\begin{tabular}{|c|c|c|c|c|c|c|c|c|c|c|}
\hline Laws & $\Omega$ & $\theta$ & $\lambda$ & $\gamma$ & $\eta$ & $e_{1}$ & $e_{2}$ & $e_{3}$ & $e_{4}$ & Note \\
\hline \multirow[t]{2}{*}{ I } & $D_{r}$ & 1 & 1 & 1 & $d_{1}$ & 1 & 0 & 0 & 0 & \multirow{2}{*}{$\begin{array}{c}\text { Law with } \\
\text { beginning [7]. }\end{array}$} \\
\hline & $\mathrm{D}$ & 1 & 1 & $\eta$ & $d_{2}$ & 1 & 0 & 0 & 0 & \\
\hline \multirow[t]{2}{*}{ II } & $D_{r}$ & 1 & 1 & $\mu_{0}$ & $d_{1}$ & 0 & 1 & 0 & 0 & \multirow{2}{*}{$\begin{array}{c}\text { Law of polygon } \\
{[4] .}\end{array}$} \\
\hline & $\mathrm{D}$ & 1 & 1 & 0 & $d_{2}$ & 0 & 1 & 0 & 0 & \\
\hline III & $\mathrm{D}$ & 1 & 2 & 1 & $\eta$ & 0 & 0 & 0 & 0 & $\begin{array}{c}\text { Law of hyperbolic } \\
{[4] \text {. }}\end{array}$ \\
\hline IV & $\mathrm{D}$ & 1 & 2 & 2 & $\eta$ & 0 & 0 & 0 & 0 & Xeeg Law [5]. \\
\hline $\mathrm{V}$ & $\mathrm{D}$ & 1 & 1 & 0 & $\eta$ & 0 & 0 & 0 & 0 & Dersi Law $[18,4]$. \\
\hline \multirow[t]{2}{*}{ VI } & $D_{r}$ & 1 & 1 & $\xi$ & $d_{1}$ & 0 & 1 & 0 & 0 & \multirow{2}{*}{$\begin{array}{c}\text { Law of curvilinear } \\
{[16] .}\end{array}$} \\
\hline & $\mathrm{D}$ & 1 & 2 & 1 & $d_{2}$ & 0 & 1 & 0 & 0 & \\
\hline \multirow[t]{2}{*}{ VII } & $D_{r}$ & $\theta$ & 1 & 1 & $d_{1}$ & 1 & 0 & 0 & 0 & \multirow[t]{2}{*}{ Law to $[18,19]$. } \\
\hline & D & 1 & 1 & $\eta$ & $d_{2}$ & 1 & 0 & 0 & 0 & \\
\hline \multirow[t]{2}{*}{ VIII } & $D_{r}$ & 1 & 1 & 0 & $d_{1}$ & 0 & 0 & 1 & 0 & \multirow[t]{2}{*}{ Law to [6]. } \\
\hline & $\mathrm{D}$ & 1 & 1 & $\eta$ & $d_{2}$ & 0 & 0 & 1 & 0 & \\
\hline \multirow[t]{2}{*}{ IX } & $D_{r}$ & 1 & 1 & $\overline{\mu_{0}}$ & $d_{1}$ & 0 & 1 & 0 & 0 & \multirow[t]{2}{*}{$\begin{array}{l}\text { Law of curvilinear } \\
\text { version [16]. }\end{array}$} \\
\hline & $\mathrm{D}$ & 1 & 1 & 2 & $d_{2}$ & 0 & 1 & 0 & 0 & \\
\hline $\mathrm{X}$ & $\mathrm{D}$ & $\alpha+1$ & 1 & 0 & $\eta$ & 0 & 0 & 0 & 0 & Law of degree [18]. \\
\hline $\mathrm{XI}$ & $\mathrm{D}$ & 1 & 2 & 2 & $\eta$ & 0 & 0 & 0 & 0 & Christons law [8]. \\
\hline \multirow{3}{*}{ XII } & $\mathrm{D}_{1}$ & 1 & 2 & 2 & $\mathrm{~g}_{1}$ & 0 & 0 & 0 & 1 & \multirow{3}{*}{$\begin{array}{c}\text { Law of structure } \\
{[1,13] .}\end{array}$} \\
\hline & $\mathrm{D}_{2}$ & 1 & 1 & $\xi_{2}$ & $\mathrm{~g}_{2}$ & 0 & 0 & 0 & 1 & \\
\hline & $\mathrm{D}_{3}$ & 1 & 1 & 0 & $\mathrm{~g}_{3}$ & 0 & 0 & 0 & 1 & \\
\hline \multirow{3}{*}{ XIII } & $D_{3}$ & 1 & 2 & 1 & $\eta$ & 0 & 0 & 0 & 1 & \multirow{3}{*}{$\begin{array}{l}\text { Law of structure } \\
\text { version [15]. }\end{array}$} \\
\hline & $\mathrm{D}_{1}$ & 1 & 1 & $\xi_{2}$ & $\mathrm{~g}_{2}$ & 0 & 0 & 0 & 1 & \\
\hline & $\mathrm{D}_{2}$ & 1 & 1 & 0 & $\mathrm{~g}_{3}$ & 0 & 0 & 0 & 1 & \\
\hline
\end{tabular}

Table 1 
Where $\quad d_{1}=\eta(\eta<1), d_{2}=\eta(\eta>1)$,

$$
g_{1}=\eta \beta\left(\eta \beta>\beta_{1}\right), g_{2}=\eta \beta\left(\beta_{1}<\eta \beta<\beta_{2}\right), g_{3}=\eta \beta\left(\eta \beta<\beta_{1}\right) .
$$

Value $e_{1}, e_{2}, e_{3}, e_{4}$ are specifies the conditions at the boundaries of disturbances and also at the boundaries of the zones based on the considered filtration law, $\xi, \xi_{1}, \xi_{2}, \beta_{1}, \beta_{2}, \beta_{3}$ determines the coefficients at critical pressure gradients.

Problem (1)-(11) coefficients and parameters $k(x), \mu, M(x, t, u), f(x, t)$ known variables and functions that describe the characteristics of the environment fluid

$[4,5,16,19,22]$, in addition

$$
\Omega=D_{r} \cup D, D=\bar{\Omega}-\sum_{r=0}^{n} D_{r}, D_{r}=\left\{x: \overline{l_{r}}<x<l_{r}^{+}\right\}, \bar{D}=\left\{x: x_{0} \leq x \leq x_{L}\right\}
$$

$a_{1}, a_{2}, b_{1}, b_{2}$ are coefficients giving the possibility of considering mixed boundary conditions at the edges of the domain.

$$
\begin{gathered}
f(x, t)=a_{0} \sum_{r-1}^{n-1} q_{r} \delta\left(x-x_{r}\right) \\
\left\{a_{1}, a_{2}\right\}=\{1,0\},\left\{b_{1}, b_{2}\right\}=\{0,1\}, a_{0}-\text { correction factor, } \\
D_{3}=\left\{x: \bigcup_{r}\left(R_{2 r}^{+}<x<R_{2(r+1)}^{-}\right)\right\} . u=\{u ; v ; \vartheta\}
\end{gathered}
$$

if $x=\left\{D_{1} ; D_{2} ; D_{3}\right\}$ respectively $\varphi_{0}\left(x_{0}, t\right)$ and $\varphi_{2}\left(x_{0}, t\right)$ will take values depending on the parameters $a_{1}, b_{1}$ and $a_{2}, b_{2}$ and if $a_{1} \neq 0$ in this $\varphi_{0}=q_{0}, b_{1} \neq 0$ and $\varphi_{0}=u_{0}$, and also at $a_{2} \neq 0$ from $\varphi_{L}=q_{1}, b_{1}, b_{2} \neq 0$ to $\varphi_{L}=u_{0}$,

Problem (1) - (12)-In the general case, nonlinear and analytical solutions are practically impossible; therefore, numerical methods are used to solve it. The sequence of application of these methods in view of the requirements for the volume of work is given in a descriptive form since they themselves are the topic of a separate article. First, the method of iteration is applied concerning nonlinear terms of equations. Then the flow [20] is introduced, and problem (1) - (11) is written concerning the flow as a boundary value problem. The resulting equation is integrated on the segments $\left[x_{i-\frac{1}{2}}, x_{i+\frac{1}{2}}\right]$, and simultaneously the properties of the mean integral are used. In this case, the method of straight lines is used for the time derivative. Stream expressions integrate on $\left[x_{i-1}, x_{i}\right]$, and

after the corresponding statement and simplifications, we get [16, pp. 63-67] a difference boundary value problem with respect to the flow [21].

Using the connection between the flow and the desired function, formulas for the sweep coefficients are obtained. The values of the sweep coefficients, as well as the flow and the required functions at the edges of the region, are determined from the boundary conditions of the problem. 
In this case, formulas are written to determine the position of the unknown boundaries of disturbances and the moving boundaries of the zones. The iteration method is also used for unknown boundaries. A short algorithm for calculating the difference-iterative problem is as follows:

1) The forward running coefficients are calculated;

2) The position of the unknown boundaries of disturbances is determined.

3) Using the found positions of the perturbation boundaries as the initial one, the execution of points 1 and 2 is repeated, etc.

4) The iteration conditions for the boundaries are checked if it is satisfied then:

5) The value of the sought functions is calculated, and, if necessary, the flow in the opposite direction and the iteration conditions are checked both for the source function and for the flow. If it is done, then it goes to the second time step; if not, the calculation procedure is repeated again;

6) The iterated value from the previous iteration is taken as the initial iteration.

The above sequence is computed true for problems with unknown boundaries. Here we briefly recall that the original multiparameter initial-boundary value problem includes 13 different laws and calculation algorithms based on which group belongs to a particular mathematical model since the rules of computational processes are different.

Let us first group these laws of outcome from the proximity and similarity of the organization of the computational process. In the first group, we will include laws I, VII, VIII since they are characterized by the existence of disturbed and undisturbed regions. The second group includes laws II, VI, IX, XII, XIII, where the entire filtration area is covered by the disturbance, with unknown moving boundaries between different filtration zones. The third group contains laws III, IV, V, X, XI. There are no unknown boundaries of disturbances for them, and each of them contains an individual characteristic nonlinearity. Law $\mathrm{V}$ is used to determine the comparability of other laws used in the initial iteration.

The sequence of calculations for these groups is different, and following this, multiparameter computational algorithms are built, taking into account these phenomena. For the computational procedure, a table was also developed similarly to Table 1, where the coefficients and parameters are taken based on belonging to one or another group.

\section{Results and Discussion}

To illustrate the computational feasibility of the developed algorithms, we present fragments of calculations for the following test data is located at the point $x_{0}=0$.

$$
\begin{aligned}
& k=0.8, \beta=0.01, u_{0}=1, L=4000 \mu, \Delta \tau=0.00675 ; h=25 \cdot 10^{-3}, q_{0}=0.0864, \text {, the well } \\
& v=1, M=1
\end{aligned}
$$

The results are partially for the VI law given in Table 2, which shows the values of the function for different and the corresponding positions of the perturbation boundary.

Table 2.

\begin{tabular}{|c|c|c|c|c|}
\hline $\mathrm{x}$ & $10^{-2}$ & $10^{-1}$ & $3 \cdot 10^{-1}$ & $7 \cdot 10^{-1}$ \\
\hline 0 & 0.98987 & 0.96770 & 0.94140 & 0.90516 \\
0.1 & 0.99628 & 0.97609 & 0.95050 & 0.91441 \\
0.5 & 0.99986 & 0.99300 & 0.97558 & 0.94275 \\
0.9 & 0.99999 & 0.99728 & 0.98606 & 0.95883 \\
1 & 0.99999 & 0.99740 & 0.98912 & 0.96010 \\
\hline$l(t)$ & 0.23 & 0.68 & 0.85 & 0.98 \\
\hline
\end{tabular}


To determine the propagation rate of the boundaries of disturbances between small and large regions of the disturbance, the same problem was solved for $q_{0}=0$ and the well at point $x_{1}=0,5$. $q_{1}=0.1728$, initial disturbance boundary $l_{1}^{(0)}=0.4839$ (it is calculated using the perturbation $l_{1}^{(0)}=x_{1}-l_{1}^{(0)} \cdot \frac{q_{1}}{2}$ ). Excerpts of the rate of advancement of the left and right boundaries are shown in

Table 3.

Table 4.

\begin{tabular}{|c|c|c|c|c|c|}
\hline$t$ & $l_{1}^{-}$ & $l_{1}^{+}$ & $\begin{array}{l}\text { The } \\
\text { laws }\end{array}$ & $675 \cdot 10^{-5}$ & $675 \cdot 10^{-4}$ \\
\hline $1 \cdot 10^{-3}$ & 0.42 & 0.58 & I & 0.98133 & 0.81330 \\
\hline $5 \cdot 10^{-3}$ & 0.33 & 0.67 & VI & 0.98502 & 0.82670 \\
\hline $1 \cdot 10^{-2}$ & 0.27 & 0.73 & II & 0.98616 & 0.82975 \\
\hline $5 \cdot 10^{-2}$ & 0.09 & 0.91 & III & 0.98910 & 0.83280 \\
\hline $8 \cdot 10^{-2}$ & 0.00 & 1.00 & $\mathrm{~V}$ & 0.99016 & 0.83415 \\
\hline
\end{tabular}

Shows the average values of the function $u(x, t)$ for some selected filtration laws

$$
\left(u_{c p}=\int_{0}^{L} u(x, t) d x\right) \text {. }
$$

Table 5.

\begin{tabular}{|l|c|c|c|c|c|}
\hline & 0 & 0.1 & 0.2 & 0.3 & 0.4 \\
\hline $\mathrm{V}$ & 0.7984 & 0.7950 & 0.7859 & 0.7710 & 0.7505 \\
& & & & & \\
\hline III & 0.9033 & 0.8793 & 0.8392 & 0.7855 & 0.7209 \\
\hline I & 1 & 0.9800 & 0.8931 & 0.7615 & 0.6634 \\
\hline IV & 0.8812 & 0.8626 & 0.8290 & 0.7841 & 0.7382 \\
\hline IX & 0.9622 & 0.9214 & 0.8716 & 0.8051 & 0.7612 \\
\hline
\end{tabular}

Table 6. The rate of change of the left and right boundaries of disturbances

\begin{tabular}{|c|c|c|c|}
\hline $\mathrm{X}$ & $675 \cdot 10^{-6}$ & $135 \cdot 10^{-5}$ & $337 \cdot 10^{-5}$ \\
\hline$l_{1}^{-}$ & 0.186 & 0.166 & 0.104 \\
\hline$l_{1}^{+}$ & 0.314 & 0.334 & 0.370 \\
\hline$l_{2}^{-}$ & 0.436 & 0.416 & 0.370 \\
\hline$l_{2}^{+}$ & 0.564 & 0.584 & 0.630 \\
\hline$l_{3}^{-}$ & 0.686 & 0.666 & 0.630 \\
\hline$l_{3}^{+}$ & 0.814 & 0.834 & 0.8965 \\
\hline
\end{tabular}

Table 6 shows the rate of change of the left and right boundaries of disturbances for different steps $t$ when three wells are located inside the region at points: 
$x_{1}=0.25 ; x_{2}=0.50 ; x_{3}=0.75$ with debit $q_{1}=q_{2}=q_{3}=0.8888$, at zero approximations

of the perturbation boundaries $e_{1}^{-\frac{(0)}{1}}=0.2488 ; e_{2}^{-\frac{(0)}{2}}=0.4987 ; e_{3}^{-\frac{(0)}{-}}=0.7487$

shows the results of calculating the values of the functions to the left of when at the points where the source is located for

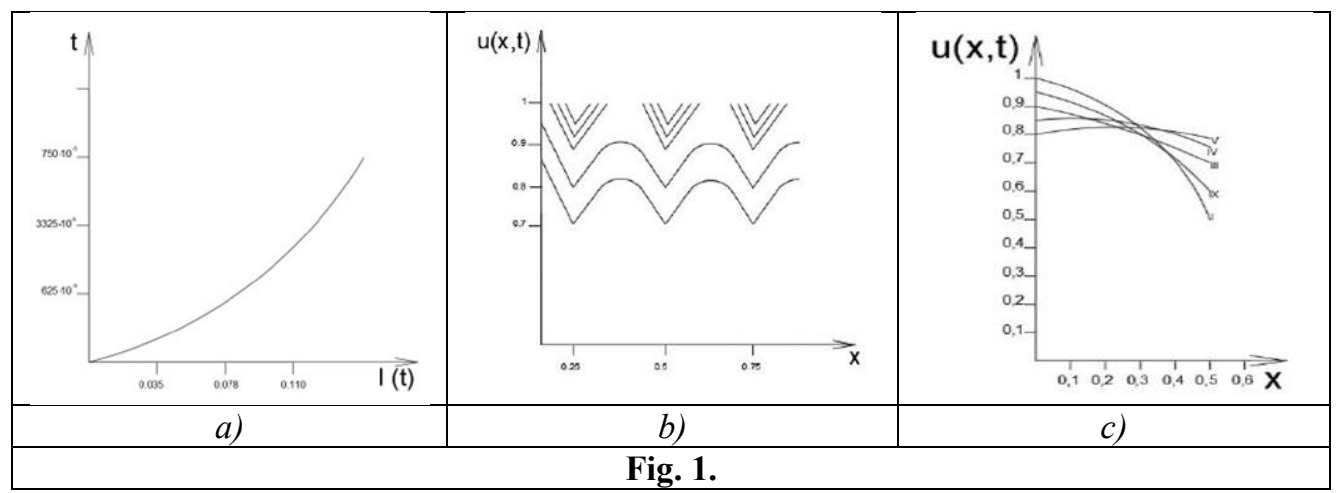

Figure $1 \mathrm{a}$, shows the time curve of the disturbance boundary.

If there are three wells in the area at points $x_{1}=0.25, x_{2}=0.5, x_{3}=0.75$ then the profile of the change in the function and the perturbation boundary under the law $\mathrm{I}$ is shown in Figure 1, b.

Function change curves $u(x, t)$ for various filtration laws is shown in Figure c.

It can be seen from the graph that the curve of the function change for laws III, IV, IX lies between the $\mathrm{V}$ and I laws, and at the same time, the minimum pressure at the bottom of the source will be at law I.

\section{Conclusions}

In conclusion, it should be noted that the constructed multi-parametric model reflecting the existing laws of fluid filtration is a compact, economical and form-based representation of mathematical models. The developed model and computational algorithms can be used to calculate the technical and economic indicators of the development of fluids in porous media. The model is based on the form of openings, and when new laws of filtration appear, they can be additionally included in this structure.

It should be noted that during the iteration, to determine the position of the left and right boundaries of the point source perturbations, one should use a two-way iteration or the method of "shuttle" iterations $[16,22]$ which allows you to quickly determine the position of the boundary.

\section{References}

1. Levashkevich V.G, Dependence of the viscosity, mobility and filtration rate of anomalously viscous oil on the pressure gradient, Izvestiya VUZov series "Oil and Gas"11 pp. 58-63. (1982)

2. Khamdamov R, Kayumov Sh.Sh. On some problems arising in the mathematical modeling of filtration problems for structured fluids. Collection of scientific papers of 
the republican scientific and practical conference of young scientists. Tashkent., (1), pp. 39-41. (1997)

3. Devlikamov V.V, Khabibulin Z.A, Kabirov M.M. Abnormal oil. M .: Nedra.. p.168s. (1975).

4. Molokovich Yu.M, Skvortsov E.V. One-dimensional filtration of a compressible visco-plastic fluid. Kazan: Publishing house of KGU, p. 64. (1971).

5. Heer B, Hefner F, On the formation of a nonlinear filtration law and on the numerical solution of a multiphase incompressible fluid. Novosibirsk: Computing Center of the Siberian Branch of the USSR Academy of Sciences. pp. 315-318. (1975).

6. Alishaev M.G, Vakhitov G.G, Gekhtman M.M, Grushov I.F. Some features of filtration of reservoir Devonian oil at low temperatures, Izv. Academy of Sciences of the USSR. MGH. (3), pp. 166-169. (1966).

7. Mirzazhanzade A.Kh. Problems of hydrodynamics of viscous - plastic and viscous fluids in oil production. Baku. Aznefteizdat. pp 360. (1959).

8. Khristianovich S.A. Groundwater movement does not follow Darcy's law, PMM. 4. (1), pp. 33-52. (1940)

9. Mukhidinov N.M. Application of the method of straight lines for the numerical solution of some problems in the theory of filtration of viscous - plastic fluids (VPL), Questions of computational and applied mathematics. Issue 13. Tashkent. RISO AN UzSSR, pp. 121-143. (1972).

10. Markelova A.M, Trifonov A.I, Olkhovskaya V.A. Two-phase filtration model for nonlinear viscoplastic oil in the presence of an active aqueous phase. Collection of scientific papers of the I international scientific conference of young scientists. 2. Novosibirsk., pp. 251-253. (2014).

11. Pritchin G.P, Krevsky G.G, Gerasimov D.S, Zhuravlev A.A. Structural behavior of non-Newtonian fluids. Materials of the international scientific and technical conference. T. 1. Tyumen. TyumGNGU, pp. 270-272. (2011).

12. Gilmiyev D.R. Numerical study of the filtration of non-Newtonian fluids in a porous medium. Materials of the international scientific and technical conference. T. 1. Tyumen. TyumGNGU., pp. 177-181. (2011).

13. Kayumov Sh. On the question of mathematical modeling of structured fluids. Proceedings of the international conference RDAAM-2001. 6, (2). Novosibirsk., pp. 183-190. (2001).

14. Kayumov Sh, Iskanadzhiev I, Narziev A. Constructed multiparameter mathematical models of the problem of the theory of filtration of unstructured and anomalously structured fluids (gas, gas condensate, oil and water). Materials of the International Scientific and Technical Conference "Oil and Gas of Western Siberia" v. 1. Tyumen, pp. 211-215. (2011).

15. Kayumov Sh. Multiparameter mathematical models of the problem of the theory of filtration of structured and anomalously structured fluids. Bulletin of Tashkent State Technical University. 4, pp. 20-24. (2010)

16. Kayumov Sh. Approximate analytical methods for solving problems of the theory of filtration of viscoplastic fluids. Tashkent. FAN Publishing House. p. 156. (1991).

17. Kayumov Sh, Mustapakulov Kh.Ya. Mathematical models of three-dimensional problems of the theory of fluid filtration with nonlinear effects. Materials of the IV International Scientific and Practical Conference Actual problems of science and education in a modern university. Sterlitimak, Russia, pp. 114-120. (2019)

18. Aliev V.A, Gurbanov R.S, Mamedov G.A, Farzane Ya.G. Generalized Darcy's law. Proceedings of AzINEFTEKHIM. 26. Baku., pp. 29-33. (1967).

19. Barenblatt G.I. Entov V.M, Ryzhik V.M. Theory of non-stationary filtration of liquid and gas. Moscow: Nedra, p.288. (1972). 
20. Degtyarev L.M, Favorsky A.P. The streaming version of the sweep method for difference problems with a strongly varying coefficient, Journal of Computational Mathematics and Mathematical Physics.. 9. 2, pp. 211-217. (1969).

21. Samarskiy A.A, Gulin A.V. Numerical methods. M. Science. p 484. (1989).

22. Kayumov Sh. Mathematical modeling of the problem of filtration theory with free boundaries. Tashkent. TGTU. p. 274. (2017). 